\title{
Lidil
}

Revue de linguistique et de didactique des langues

\section{Bange Pierre (en collaboration avec Rita Carol et Peter Griggs)}

2005, L'apprentissage d'une langue étrangère, Paris, L'Harmattan

(« sémantiques »).

Marinette Matthey

\section{CpenEdition}

Journals

Édition électronique

URL : http://journals.openedition.org/lidil/36

DOI : 10.4000 /lidil.36

ISSN : 1960-6052

Éditeur

UGA Éditions/Université Grenoble Alpes

Édition imprimée

Date de publication : 1 décembre 2006

ISBN : 2-914176-15-5

ISSN : $1146-6480$

Référence électronique

Marinette Matthey, «Bange Pierre (en collaboration avec Rita Carol et Peter Griggs) », Lidil [En ligne], 34 | 2006, mis en ligne le 01 octobre 2007, consulté le 21 septembre 2020. URL : http://

journals.openedition.org/lidil/36 ; DOI : https://doi.org/10.4000/lidil.36

Ce document a été généré automatiquement le 21 septembre 2020.

(C) Lidil 


\title{
Bange Pierre (en collaboration avec Rita Carol et Peter Griggs)
}

\author{
2005, L'apprentissage d'une langue étrangère, Paris, L'Harmattan
}

(« sémantiques »).

\section{Marinette Matthey}

1 Pierre Bange, Rita Carol et Peter Griggs travaillent en équipe depuis de nombreuses années dans le domaine cognition et interaction de la linguistique de l'acquisition. Le livre publié chez l'Harmattan donne une vision synthétique de leurs travaux, qui allient réflexion théorique (modèle cognitif de l'apprentissage d'une L2) et contextualisation sous forme d'analyses de cas.

2 La première partie, rédigée par Pierre Bange, s'ouvre sur une explicitation des conceptions linguistiques des auteurs. Conception pragmatique tout d'abord (que P. Bange a déjà présentée dans son ouvrage «Analyse conversationnelle et théorie de l'action $\left.{ }^{1} »\right)$ et, pour ce qui concerne l'aspect psycholinguistique, conception fondée sur les travaux de Levelt qui envisage la production langagière comme une suite de «moments" (intention communicative - planification d'un énoncé - encodage grammatical et phonologique - articulation). À l'issue de ce premier chapitre, P. Bange conclut, contre Chomsky, qu'il n'y a pas de faculté de langage indépendante de la pensée : « la coordination des actions humaines n'est possible que parce que la pensée (outil individuel) et la langue (outil collectif) sont dans une relation d'étroite interdépendance » (p. 29).

3 Le deuxième chapitre présente le même travail d'explicitation et de mise en relation de notions issues des domaines cognition et interaction, cette fois sur les conceptions de l'apprentissage. Anderson est convoqué pour établir la différence entre savoir procédural et savoir déclaratif et établir que le langage est un savoir procédural (p. 36). Le concept d' étayage de Bruner, envisagé comme "une modalité interactionnelle» (p. 49), est également introduit pour rendre compte de la possibilité même des apprentissages langagiers. Cognition et interaction sont donc inséparables : «La préoccupation du didacticien (maximiser l'apprentissage de la langue) ne doit pas conduire à sousestimer ou même à ignorer que l'interaction a un enjeu social et que cet enjeu est 
premier, car cela conduirait à sous-estimer ou ignorer la condition de tout apprentissage procédural : l'action, l'action conjointe, l'interaction, dans laquelle les deux partenaires sont égaux. L'apprentissage de la communication ne peut être assuré et ne peut être facilité que dans la communication. Mais il ne peut être réalisé que par celui qui doit apprendre, par son propre travail cognitif. Le tuteur n'est tuteur qu'au regard de l'incapacité linguistique du novice. Il ne l'est pas au regard des actions où la langue intervient comme un moyen et dans lesquelles les interactants sont égaux et autonomes. L'interaction est la condition de l'apprentissage, elle n'en est pas la réalité. Si elle veut progresser, la didactique doit marcher sur ses deux jambes » (p. 55).

4 Le chapitre 3 introduit la question des différences entre l'acquisition de L1 et de L2. P. Bange se livre tout d'abord à un commentaire de différents extraits de Vygotski puis propose une bipartition entre conditions internes et externes de l'apprentissage, pour aboutir à l'exposé des principes d'une didactique des langues fonctionnelle, c'est-à-dire une didactique qui s'appuie sur les deux «jambes» que sont les caractéristiques intrinsèques de la communication humaine d'une part, de la cognition de l'autre.

5 La deuxième partie réunit des études empiriques, signées des trois auteurs pour les trois premières, par Rita Carol pour la dernière. Le chapitre 4 reprend et reformule un article déjà paru dans Marges linguistiques 4 (2002). Les données sont issues du corpus de thèse de Rita Carol et portent sur la construction progressive des formes verbales du parfait en allemand L2. Les principes d'analyse procédurale partent de la description/ simulation des règles de l'interlangue de l'apprenant, exprimées en tenant compte de ce qu'on pourrait appeler l'intentionnalité grammaticale du locuteur, par des règles $\mathrm{du}$ type si... alors. Par exemple : «si tu veux marquer formellement le passé du verbe gehen, alors a) utilise l'auxiliaire sein à la forme conjuguée correspondante ; b) utilise la forme non marquée gehen». Un schéma formel relativement complexe (modèle cognitif) permet de rendre compte du cheminement procédural durant la période considérée (6 mois).

Le chapitre 5 propose une analyse d'une leçon d'un manuel d'allemand langue étrangère et de l'interaction en classe suscitée par une des activités proposées. Les données présentées permettent de montrer à quel point les activités induites par les tâches du manuel correspondent à des conceptions de l'apprentissage périmées en regard du modèle qui a été présenté dans les chapitres précédents.

7 Le chapitre 6 propose une critique en règle de l'approche communicative, comprise comme un xème avatar des conceptions béhavioristes de l'apprentissage (imitation, exercices, automatisation). Il contient également la présentation d'une unit d'une méthode d'anglais pour adultes, dans laquelle on observe une réorientation cognitive de la perspective communicative, c'est-à-dire que les activités proposées se basent sur une double conception des langues et de leur apprentissage tout à fait compatibles avec les principes théoriques interactionnels et cognitifs illustrés et défendus par les auteurs tout au long de leur ouvrage.

8 Finalement, Rita Carol, dans le septième chapitre, analyse des interactions dans une classe de CP bilingue en Alsace. Les élèves (L1 français) apprennent à faire des additions avec retenue en allemand L2. Ce chapitre permet d'illustrer de la manière la plus convaincante qui soit l'intrication entre construction de savoir mathématique et linguistique et fait apparaitre l'immersion comme «la bonne forme» de l'enseignement des L2, compatible avec l'état des connaissances scientifiques (linguistiques et psycholinguistiques) actuelles. 
Une post-face originale donne à lire un échange de courriels entre Pierre et Jacques Bange (frère de l'auteur, professeur de russe L2), qui réagit à une version du manuscrit. Cet échange est une bonne illustration des points de vue finalement pas si antithétiques du chercheur didacticien (à qui l'on reproche parfois de ne pas connaitre de l'intérieur la réalité qu'il décrit) et de l'enseignant, qui découvre des références théoriques linguistiques et psychologiques jetant une autre lumière sur une réalité qu'il connait parfaitement. Le livre s'achève donc sur une note d'espoir pour les rapports entre didactique et enseignement !

10 Le couplage de deux univers de référence (action interaction pragmatique d'un côté ; psycholinguistique cognitive de l'autre) n'est pas toujours facile à réaliser, et les interactionistes reprocheront certainement aux trois auteurs une conception «faible » de l'interaction, alors que les cognitivistes pourront souligner le caractère un peu daté des références. Pour les premiers, envisager l'interaction comme condition des apprentissages et non comme formatant les connaissances, n'est pas suffisant car le travail cognitif de l'apprenant est encore vu comme quelque chose d'individuel et non comme une activité située et distribuée. La question de la mise en rapport du "dehors" (l'interaction communicative) et du «dedans " (l'évolution du traitement procédural du langage) est finalement vue, dans cette contribution des trois chercheurs, comme dépendant uniquement des intentions du locuteur-apprenant, qui résout certes des tâches communicatives et grammaticales en collaboration avec autrui, mais qui gère seul son apprentissage.

11 Pour les seconds (les cognitivistes), modaliser le fonctionnement cognitif par des règles procédurales de type si... alors apparaitra peut-être comme une vision trop calquée sur le raisonnement logique. Ce type de règles formelles est très puissant; elles permettent de rendre compte de la logique des formes de l'interlangue de l'apprenant à un moment donné de son développement ( $c f$. ci-dessus l'exemple de l'évolution de la référence au passé en allemand), mais également des conduites des élèves face à un exercice : Si tu veux réussir l'exercice, alors a) analyse le modèle b) reproduis le résultat de ton analyse dans chaque phrase. Peut-on vraiment assimiler dans un même modèle descriptif et explicatif ce qui relève de la planification d'un énoncé et ce qui relève des conduites face à un exercice scolaire? La question reste ouverte.

12 Comme restent ouvertes (heureusement!) les questions autour des conceptions de l'apprentissage: on lit, p. 86 « Tout apprentissage commence par un stade déclaratif: l'apprentissage de faits et de concepts »; mais aussi, p. 153 « Nous ne pensons pas qu'on apprend d'abord une langue, et notamment ses règles de grammaire, et que cela permet ensuite de la pratiquer ». N'y a-t-il pas une certaine contradiction entre ces deux affirmations? Contradiction qui reflète bien la mobilité des conceptions de l'apprentissage même chez les spécialistes !

13 Dans la même veine, il semble aussi que les auteurs s'inscrivent fortement contre la théorie béhavioriste, notamment parce qu'elle accorde une large place à l'imitation, ce qui est une conception cognitive naïve, selon eux. Or Vygotski, auquel les mêmes auteurs se réfèrent largement, accorde aussi un rôle important à l'imitation dans les apprentissages (il considère même que l'imitation est un signe observable de la zone du prochain développement (zone proximale)). Comment concilier ces deux visions de l'imitation? Un chapitre d'explicitation sur cette question aurait été bienvenu.

14 Au-delà de ces quelques remarques critiques, ce livre met un point d'orgue à un programme de recherche mené par nos trois auteurs sur une durée de près de 15 ans, 
programme qui se caractérise par sa consistance et sa rigueur. Il répondra aux besoins des enseignants intéressés par les théories sur les langues, le langage et leur apprentissage. Il propose également une vision des rapports entre interaction et cognition qui intéressera les didacticiens.

NOTES

1. Bange, P. (1992) : Analyse conversationnelle et théorie de l'action, Paris, Didier (LAL).

\section{AUTEUR}

MARINETTE MATTHEY

Université Stendhal Grenoble3, Lidilem 